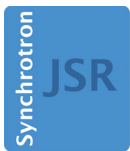

JOURNAL OF

SYNCHROTRON

RADIATION

ISSN 1600-5775

Received 10 July 2014

Accepted 29 April 2015

Edited by I. Schlichting, Max Planck Institute for Medical Research, Germany

Keywords: phase-contrast computed tomography; computed tomography reconstruction.

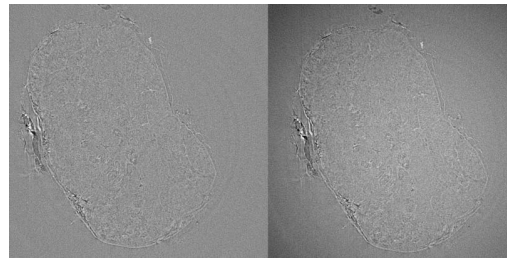

C 2015 International Union of Crystallography

\section{Assessment of freeware programs for the recon- struction of tomography datasets obtained with a monochromatic synchrotron-based X-ray source}

 \\ Murray Pettitt, ${ }^{\mathrm{e}}$ Rajni Chibbar, ${ }^{\mathrm{f}}$ Seyedali Melli ${ }^{\mathrm{g}}$ and James Montgomery ${ }^{\mathrm{h}}$.
}

\author{
${ }^{a}$ College of Agriculture and Bioresources, University of Saskatchewan, 51 Campus Drive, Saskatoon, SK, Canada \\ S7N 5B4, 'bSmall Animal Clinical Sciences, Western College of Veterinary Medicine, University of Saskatchewan, \\ 52 Campus Drive, Saskatoon, SK, Canada S7N 5B4, 'Department of Medical Imaging, University of Saskatchewan, \\ 103 Hospital Drive, Saskatoon, SK, Canada S7N 0W8, dVeterinary Biomedical Sciences, Western College of Veterinary \\ Medicine, University of Saskatchewan, 52 Campus Drive, Saskatoon, SK, Canada S7N 5B4, ${ }^{\mathbf{e} C o l l e g e}$ of Agriculture and \\ Bioresources, University of Saskatchewan, 51 Campus Drive, Saskatoon, SK, Canada S7N 5B4, 'Pathology and Lab \\ Medicine, College of Medicine, University of Saskatchewan, 103 Hospital Drive, Saskatoon, SK, Canada S7N 0W8, \\ ${ }^{\mathrm{g}}$ Department of Electrical and Computer Engineering, University of Saskatchewan, 57 Campus Drive, Saskatoon, \\ SK, Canada S7N 5A9, and h'College of Medicine, University of Saskatchewan, 103 Hospital Drive, Saskatoon, SK, \\ Canada S7N 0W8. *Correspondence e-mail: james.montgomery@usask.ca
}

Synchrotron-based in-line phase-contrast computed tomography (PC-CT) allows soft tissue to be imaged with sub-gross resolution and has potential to be used as a diagnostic tool. The reconstruction and processing of in-line PC-CT datasets is a computationally demanding task; thus, an efficient and user-friendly software program is desirable. Four freeware programs (NRecon, PITRE, H-PITRE and Athabasca Recon) were compared for the availability of features such as dark- and flat-field calibration, beam power normalization, ring artifact removal, and alignment tools for optimizing image quality. An in-line PC-CT projection dataset $\left(3751\right.$ projections, $180^{\circ}$ rotation, $10.13 \mathrm{~mm} \times 0.54 \mathrm{~mm}$ ) was collected from a formalin-fixed canine prostate at the Biomedical Imaging and Therapy Bending Magnet (BMIT-BM) beamline of the Canadian Light Source. This dataset was processed with each of the four software programs and usability of the program was evaluated. Efficiency was assessed by how each program maximized computer processing power during computation. Athabasca Recon had the least-efficient memory usage, least user-friendly interface, and lacked a ring artifact removal feature. NRecon, PITRE and H-PITRE produced similar quality images, but the Athabasca Recon reconstruction suffered from the lack of a native ring remover algorithm. The 64-bit version of NRecon uses GPU (graphics processor unit) memory for accelerated processing and is userfriendly, but does not provide necessary parameters for in-line PC-CT data, such as dark-field and flat-field correction and beam power normalization. PITRE has many helpful features and tools, but lacks a comprehensive user manual and help section. H-PITRE is a condensed version of PITRE and maximizes computer memory for efficiency. To conclude, NRecon has fewer imaging processing tools than PITRE and H-PITRE, but is ideal for less experienced users due to a simple user interface. Based on the quality of reconstructed images, efficient use of computer memory and parameter availability, H-PITRE was the preferred of the four programs compared.

\section{Introduction}

In-line phase-contrast computed tomography (PC-CT) is a micro-computed tomography (MCT) technique and has steadily become an important imaging modality for the biomedical research community (Bravin et al., 2013; Suortti \& Thomlinson, 2003; Thomlinson et al., 2005; Lewis, 1997, 2004; Zhou \& Brahme, 2008). Compared with X-ray absorption techni- 
ques, PC-CT relies on changes in the phase of X-rays as they pass through tissues, can provide spatial resolution in the micrometre range, and up to 1000 times greater image contrast than traditional computed tomography (CT) methods in the hard X-ray region (Vo et al., 2012). An important advantage over conventional X-ray modalities is that soft tissues and fine structures between tissues of different densities are readily distinguishable with in-line PC-CT. This technique has been successfully implemented using synchrotron radiation sources allowing for the qualitative collection of phase information of X-rays (Chen et al., 2010).

While there are many different reconstruction algorithms, most can be sub-classified into two main categories; filtered back-projection (FBP) and algebraic iterative algorithms (Flores et al., 2013). FBP methods are based on analytical algorithms using the inverse Fourier transform on equally spaced projections (Flores et al., 2013) while algebraic iterative methods fit predictor models to data through iterations (Deák et al., 2013) and do not require evenly spaced projections. FBP has long been the standard reconstruction method running on a central processing unit (CPU); however, with the advent of third-generation synchrotron sources and new detectors, larger datasets significantly increase reconstruction times making new high-performance computing systems mandatory (Marone \& Stampanoni, 2012). Many software programs are now making use of graphics processing units (GPUs) to increase efficiency for these demanding algorithms or for large datasets for tomographic reconstructions (Flores et al., 2013). Use of a GPU can significantly reduce the data processing time.

Here, four freeware programs (NRecon ${ }^{\mathbf{1}}$, Athabasca Recon ${ }^{\mathbf{2}}$, PITRE $^{3}$ and $H$-PITRE ${ }^{4}$ ) available for use in PC-CT data reconstruction are compared for usability, available parameters, help resources, computing efficiency and resulting image quality. These programs are based on the FBP method, but use different filtering algorithms for image reconstruction.

\section{Background information}

\subsection{Phase-contrast $\mathrm{CT}$}

In conventional X-ray imaging, image contrast arises from differences in attenuation of the X-ray beam by structures with differing linear attenuation coefficients. When imaging low- $Z$ materials with similar linear attenuation coefficients, such as biological tissue, with X-rays in the $10-100 \mathrm{keV}$ energy range, phase shift probabilities between different tissues are approximately 1000 times larger than for linear attenuation,

\footnotetext{
${ }^{1}$ NRecon, version 1.6.8.0, 2011; SkyScan (Bruker-microCT), Kontich, Belgium, http://www.skyscan.be/products/downloads.htm.

${ }^{2}$ Athabasca Recon, version 1.3; Bone Imaging Laboratory, University of Calgary, http://bonelab.ucalgary.ca/software/athabasca_recon.

${ }^{3}$ PITRE, version 3.0; R. C. Chen, Istituto Nazionale di Fisica Nucleare (INFN), Sezione di Trieste, Trieste, Italy; Sincrotrone Trieste SCpA, Trieste, Italy, http://webint.ts.infn.it/en/research/exp/beats2/PITRE.html.

${ }^{4}$ H-PITRE, beta version; R. C. Chen, Istituto Nazionale di Fisica Nucleare (INFN), Sezione di Trieste, Trieste, Italy; Sincrotrone Trieste SCpA, Trieste, Italy, http://webint.ts.infn.it/en/research/exp/beats2/PITRE.html.
}

thereby allowing visualization of structures which are not visible with conventional attenuation-based techniques (Zhou \& Brahme, 2008; Wu et al., 2008). In propagation-based phasecontrast imaging the sample is irradiated with highly spatially coherent X-rays. The detector is set a certain distance downstream from the sample such that, when the X-ray wavefront is diffracted passing through the sample, the perturbed wave interferes upon free-space propagation after the sample according to Fresnel diffraction. This gives rise to interference fringes at the edges of the different sample structures consequently enhancing the edges (Diemoz et al., 2012). PC-CT uses these shifts in phase of the X-rays to provide high contrast. Both qualitative images, with strong edge enhancement but no phase retrieval, and quantitative (with phase retrieval) images can be obtained (Chen et al., 2010). Wu et al. (2008) terms these as direct 'phase-contrast tomography' when the edgeenhancement without phase retrieval is utilized, and ' $\mathrm{X}$-ray phase tomography' where phase retrieval was performed. The direct phase-contrast tomogram is a mixture of three components [(i) the three-dimensional map of the sample's linear attenuation coefficients, (ii) the map of the threedimensional Laplacians of its refraction indices, and (iii) artifacts related to the global distribution of tissue attenuation coefficients and refraction indices], thus there is need for phase retrieval for accurate and quantitative tomography for medical applications (Wu et al., 2008).

Burvall et al. (2011) identified seven methods of in-line phase retrieval which satisfy two conditions making them applicable to tomography. First, that they require only one image at each projection angle, and second, that they utilize analytical methods rather than iterative methods to minimize the reconstruction time. Burvall et al. also provide a scheme for choosing which reconstruction method to use based on the characteristics of the data set. Phase retrieval is recognized as an important aspect of phase-contrast imaging, but was not considered a main focus of this study for two reasons: (i) of the four programs assessed, only two (PITRE, H-PITRE) are capable of performing phase retrieval, and (ii) Mohammadi et al. (2013) provide a comparison of the free software programs ANKAPhase (Weitkamp et al., 2011, 2014) and PITRE (Chen et al., 2012a, 2013a) for implementing phase-retrieval algorithms with propagation-based X-ray tomography. As such this work only reviews the performance of the three-dimensional reconstruction software. We use phase-contrast images as the input data sets, but the results are equally valid for absorption contrast images.

Synchrotrons provide a high-intensity and highly coherent $\mathrm{X}$-ray beam with parallel geometry and monochromatic energy. This allows for the properties of diffraction and interference to be exploited (Meuli et al., 2004) and avoids problems that occur with images produced using conventional polychromatic X-ray cone-beams such as low contrast, high noise and low dimensional accuracy (Kastner et al., 2010). Therefore, when in-line PC-CT is used in conjunction with synchrotron radiation, it dramatically increases the ability to image soft tissues, such as the prostate. An important advantage of the in-line PC-CT is that this method imparts low 
absorbed radiation dose to tissues (due to the use of highenergy X-rays) as compared with using only linear beam attenuation for contrast (Meuli et al., 2004). In addition, in-line $\mathrm{PC}-\mathrm{CT}$ is a useful imaging technique due to its relatively simple set up. It does not require any crystals or additional equipment besides a high-intensity high-energy monochromatic beam and a detector (Guo et al., 2012).

\subsection{Tomography algorithm}

CT is a valuable medical technique that is often used to obtain parallel cross-sectional images of the affected body part or organ. The technique involves acquiring numerous 'projection' images at angular interval steps around a sample and then using a computer-based geometric algorithm to reconstruct a view of a cross slice through the tissue (Meuli et al., 2004). The FBP method is one of the most commonly used algorithms to construct CT cross-sectional images (Herman, 2010) and makes use of the Radon transform (Radon, 1986). A projection image at a certain angle represents the line integral of the X-ray attenuation through a sample and the Radon transform of this integral represents the cross-sectional image of the sample along that path. The inverse Radon transform is applied across all the line integrals at different angular positions to reconstruct a single cross-sectional view (Kak \& Slaney, 2001). One way to represent the projection data for filtering is by using sinograms which are plots of the absorption data for the reconstruction of a single slice (Stock, 2009). Sinograms are produced by collecting the linear signal along a specific detector row in the imaging plane for all the angular views along the $180^{\circ}$ rotation and arranging them side by side (Chen et al., 2012a). FBP approximates the inverse Radon transform by a single convolution followed by a back projection of the data thus producing the cross-section image of the sample (Herman, 2010). The convolution involves the Fourier transform of the projection followed by multiplying it by a weighting function to filter the frequencies of the projections to produce higher quality reconstructions. Then the inverse Fourier transforms are summed over the image plane to back project the data over the cross-sectional plane (Kak \& Slaney, 2001). FBP is one of the most frequently used methods for it only requires one convolution integral calculation reducing computation time compared with iterative methods that approximate and improve image quality with multiple calculations. In a comparison of the adaptive statistical iterative reconstruction (ASIR) algorithm, the modelbased iterative reconstruction (MBIR) algorithm and the FBP algorithm, the MBIR method provided the best image quality of the three techniques in reducing noise and improving overall image quality (Deák et al., 2013). However, most iterative reconstruction methods require high computational power and long reconstruction times. ASIR is an exception with its reduced reconstruction time; however, it requires an initial building block of an FBP reconstructed image to accomplish this (Singh et al., 2010). Currently FBP is the algorithm used by many CT software systems and is the main algorithm used in all four software systems described here.

\section{Materials and methods}

\subsection{Data collection}

Projection data used to test these programs were collected at the Canadian Light Source (CLS) Biomedical Imaging and Therapy Bending Magnet (BMIT-BM) beamline (Wysokinski et al., 2007). This is a bend magnet beamline with a field strength of $1.354 \mathrm{~T}$. The ring energy is $2.9 \mathrm{GeV}$ and the storage ring current is a maximum of $250 \mathrm{~mA}$ operating in decay mode. The critical energy of the bend magnet source is $7.57 \mathrm{keV}$. The beamline uses a Si $(2,2,0)$ Bragg double-crystal monochronometer at a distance of $13.2 \mathrm{~m}$ from the source. An ex vivo formalin-fixed canine prostate was suspended in a plastic specimen tube within Knox Gelatine (Associated Brands Lp, Toronto, ON, Canada) positioned on a mechanical rotating stage $25 \mathrm{~m}$ from the $30 \mathrm{keV}$ monochromatic synchrotron X-ray source. A Photonic Science VHR-90 (Photonic Science, Millham, Mountfield, UK) array radiation camera (FOP coupled CCD detector, $18.67 \mu \mathrm{m} \times 18.67 \mu \mathrm{m}$ pixel size) was placed $5 \mathrm{~m}$ behind the sample to allow for free space propagation. The sample was then rotated over $180.096^{\circ}$ and 3751 projections were taken each at a rotational step size of $0.048^{\circ}$. Exposure time for each projection was $200 \mathrm{~ms}$ with total imaging time of $44 \mathrm{~min}$ with total exposure of $12.5 \mathrm{~min}$. Each projection was digitized to a $3968 \times 251$ pixel 12-bit image (TIFF greyscale format). The reconstruction software requires 16-bit TIFFs, and this change in format was made using Convert (Canadian Light Source, Inc., Saskatoon, SK, Canada). The effective pixel resolution of $12.4 \mu \mathrm{m}$ was measured by acquiring two projection images with a needle moved a known distance perpendicular to the detector on the sample stage, adding the images together, counting the number of pixels in the known distance and dividing the distance by the number of pixels. Calibration images were taken before and after the tomographic projections were completed. Further steps in processing the data depended on the software used and are described below.

\subsection{Software parameters for image calibrations and artifact removal}

In-line PC-CT reconstruction requires that certain parameters be adjusted to suit an individual dataset. The following parameters were adjusted (when available) by the four programs reviewed here: dark-field correction, flat-field correction, centre of rotation alignment, beam-hardening correction, ring-artifact correction, and intensity or beam power normalization. Calibrations or corrections to the projection files usually occur prior to conversion to sinograms or slices.

3.2.1. Dark-field and flat-field corrections. These corrections help to reduce noise. Dark current is the residual current within the detector and nearby electronics that produce small amounts of photonic radiation besides that of the source (Bourland, 2012). Dark-field calibration involves subtracting a mean dark image (averaged from all the dark images taken) from all projection images. Dark-field subtraction was completed using 20 projection images without source radia- 
tion hitting the detector (i.e. imaging with a closed shutter; ten images taken before and another ten after tomographic projections). Flat-field images are taken under the same conditions as the projections with the specimen removed and aid in subtracting the noise caused by non-uniform output from the source or the detector (Stock, 2009). Flat-field, floodfield or bright-field correction corresponds to the division by the flat-field mean image with dark-field subtraction from the projection being calibrated after dark-field subtraction (Bourland, 2012). Similar to dark-field calibration, flat-field correction was performed with averaged images created from 20 flat frames.

3.2.2. Projection alignment. Practically, it is difficult for the centre of rotation of the specimen to fall exactly at the centre of the sinograms or the projection images. Thus, the projections need to be aligned so that problems in the slice reconstruction during the FBP algorithm can be avoided. These include streaking and alias artifacts that appear, especially around very dense structures within the tissue and along edges of objects (Fig. 1). Among the methods this can be accomplished are to align the image taken at $0^{\circ}$ and the image taken at $180^{\circ}$ degrees by using their histograms (as is done by NRecon), or by adjusting the centre of the sinogram horizontally so it falls on the centre of rotation (Chen et al., 2012a).

3.2.3. Beam-hardening correction. This compensates for the lack of a linear relationship between the attenuation coefficient of the material and the material thickness when performing polychromatic X-ray CT imaging. Beam hardening can lead to image artifacts such as pronounced edges and streak artifacts unless correction is applied (Van de Casteele et al., 2002). However, for monochromatic sources, there is a linear relationship between attenuation and density of the tissue. Thus, this beam-hardening correction was ignored for the data used herein.

3.2.4. Beam power normalization. Using current imaging protocols, acquisition can require multiple hours depending on the sample size. Over time there can be slight variations in the beam intensity or power with common causes including: (i) photon source fluctuations due to temperature, e.g. the flux of a monochromator changes due to its cooling system, and (ii) decreasing flux of the synchrotron beam due to storage ring

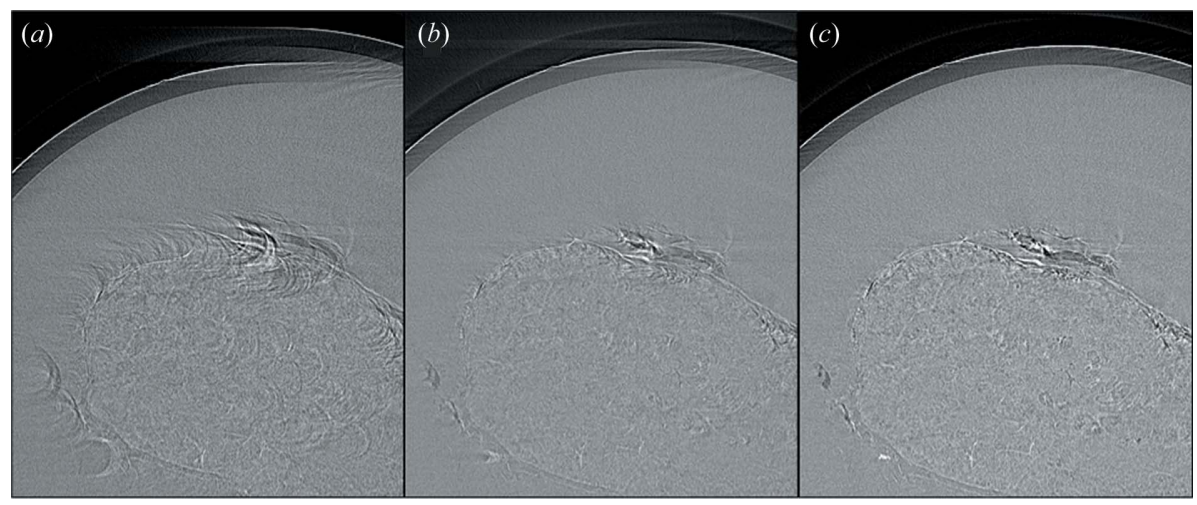

Figure 1

Previews reconstructed by PITRE. (a) Completely unaligned image, $(b)$ partially aligned image and (c) aligned image.

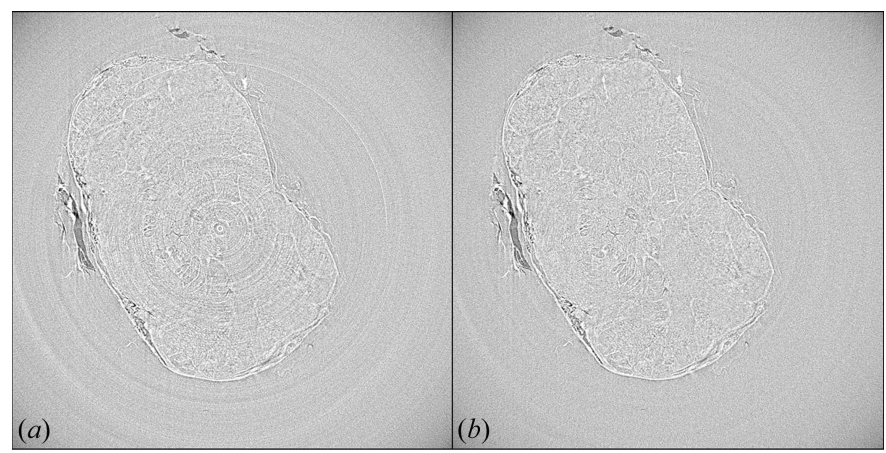

Figure 2

Reconstructed images of a canine prostate by NRecon showing images (a) without ring-removal algorithm applied and $(b)$ with ring-removal correction.

decay (Chen et al., 2012a). Therefore it may become necessary to perform a normalization calibration or beam power correction to compensate for this variation in beam intensity. The normalization procedure equalizes the intensity of the projections collected at different times. This process requires that the fluctuations are relatively homogeneous so that the intensity curve can easily be found and altered (Chen et al., 2012a). If the projections are collected over a very short time it is possible to ignore these intensity fluctuations, as their variance is minimal.

3.2.5. Ring artifact removal. Ring artifacts are concentric rings that appear around the axis of rotation in an image (Fig. 2a). They come from an invalid measurement made along the projection line that occurs in every view. This can be caused by a defective pixel on the detector, a slightly misfiltered beam, or a defective detector line parallel to the axis of rotation (Chen et al., 2013b). Dark and flat calibrations suppress, but often do not completely remove, the ring artifact due to imperfections in the dark- and flat-field images. Thus, many programs improve image quality by using a ring-removal algorithm (Bourland, 2012).

3.2.6. Efficiency. Reconstructions of high-quality CT images are extremely computationally demanding tasks (Flores et al., 2013). Recently, image processing using the GPU of a computer has become more popular as the computational performance of GPUs has surpassed that of the CPU with the advent of powerful GPUs for computer games (Eklund et al., 2013). One report showed that the use of the GPU over the CPU accelerated reconstruction with the FBP algorithm by up to 100 times (Schiwietz et al., 2006). The average amount of time spent preprocessing, reconstructing and postprocessing images was recorded for each of the four programs (Table 1).

Table 2 lists the freeware programs and their features which were investigated for their ability to deliver highquality images and complete different 
Table 1

Timing comparison for reconstruction of 3751 projections.

Comparative chart of average time spent on pre-processing and reconstructing of images with all four programs on a Gateway FX Desktop (Intel Core i72600, CPU $3.40 \mathrm{GHz}, 16 \mathrm{~GB}$ RAM, 1.5 TB HDD) with NVIDIA Quadro 6000 Video Card (384-bit memory interface, memory bandwidth $144 \mathrm{~GB} \mathrm{~s}^{-1}$ ). NRecon was the only program requiring calibration and pre-processing of the data prior to importing into the software for image reconstruction. No preprocessing of the data was required for the other three programs.

\begin{tabular}{lll}
\hline Program & $\begin{array}{l}\text { Pre-processing } \\
(\mathrm{h})\end{array}$ & $\begin{array}{l}\text { Reconstruction } \\
(\mathrm{h})\end{array}$ \\
\hline NRecon & 0.333 & $<1$ \\
Athabasca Recon & 0 & 6 \\
PITRE & 0 & 4 \\
H-PITRE & 0 & 1
\end{tabular}

facets of PC-CT reconstruction. For image quality assessment, a reference image was generated with extra post-processing with the program Ring Remover (Z. Wei, Canadian Light Source, Inc.) in MATLAB (MATLAB R2013A, MathWorks, Natick, MA, USA). The quality of each output image (Fig. 3) was assessed by calculating the peak signal to noise ratio (PSNR) and structural similarity index (SSIM). The PSNR expresses the ratio between the maximum signal value and the value of noise that can distort the quality of the image, while the SSIM provides a measure of the similarity of two images (Wang et al., 2004). Both values provide a quantitative measure of digital image quality.

\section{Results: software reviews}

\subsection{NRecon}

NRecon, version 1.6.8.0, is a freeware program developed by SkyScan [now Bruker MicroCT (Bruker-microCT, Kontich, Belgium)]. A commercial version of NRecon is available that distributes the reconstruction calculations across GPUs of many computers thereby decreasing the computation time. The no-cost version of NRecon uses the GPU of a single workstation. The program was developed to work with microCT scanners and thus lacks some features for manipulating raw data from synchrotron CT; e.g. the program does not apply the dark-field or flat-field correction to the projections, nor does it allow beam intensity normalization. The program requires 16-bit TIFF files that have been calibrated for darkfield and flat-field correction. Dark-field and flat-field corrections on our dataset were performed with a macro plugin for ImageJ (D. Cooper, College of Medicine, University of Saskatchewan, Canada). NRecon had a reconstruction time of less than $1 \mathrm{~h}$ which was comparable with H-PITRE's proces-

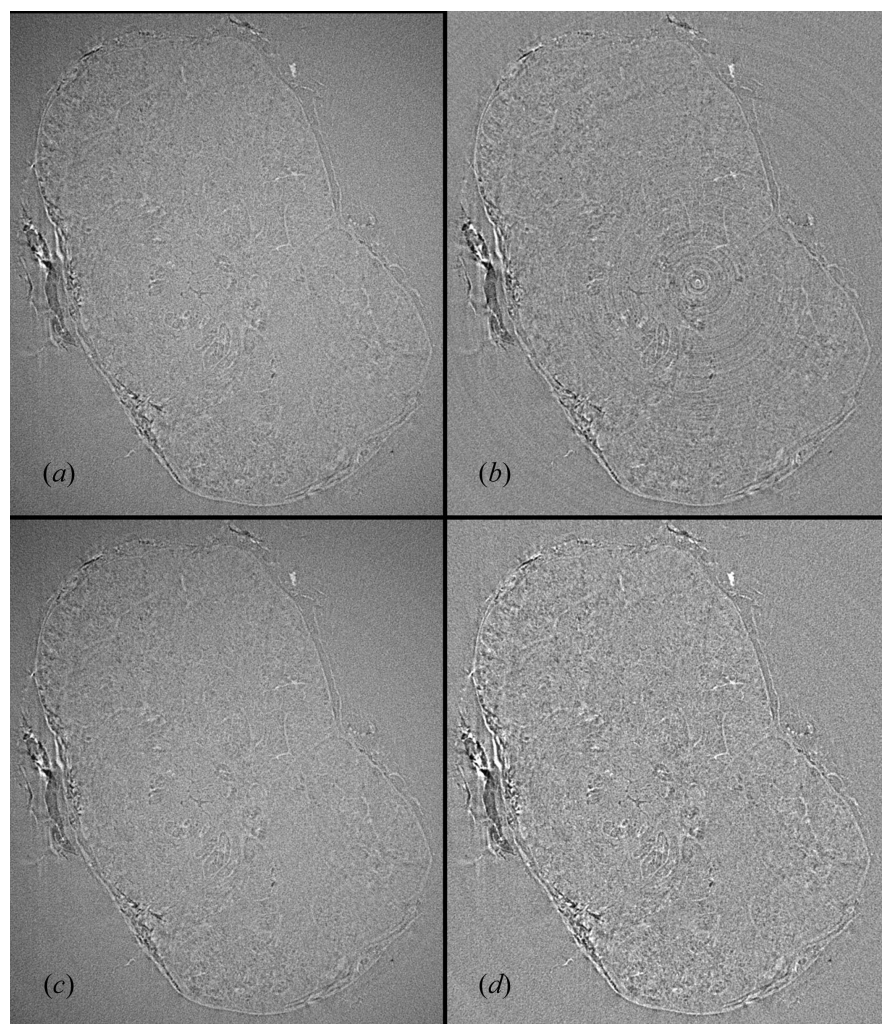

Figure 3

Output images for reconstructions performed with (a) NRecon, $(b)$ Athabasca Recon, (c) PITRE and (d) H-PITRE.

sing time, but was faster than both PITRE and Athabasca Recon. NRecon required some pre-processing, such as darkand flat-field calibration that took $20 \mathrm{~min}$, and there was no post-processing.

After uploading calibrated projection data, NRecon automatically identifies the optimized settings for projection alignment, beam-hardening correction, ring artifact correction, and smoothing filter strength. Previews of one reconstructed slice can be generated on the main page in the Start tab; the default preview is the central slice. The program approximates the post-alignment value by comparing and aligning the histograms of the first and last projections at angular positions of $0^{\circ}$ and $180^{\circ}$, respectively. The strength of the ring artifact, smoothing, beam-hardening filters and the value of the post-alignment factor can also be set manually. Final values for these parameters can be fine-tuned as the software allows the user to select from a range of previews before processing the final dataset. Fig. 2(a) shows a slice reconstructed with NRecon without ring-removal correction and Fig. 2(b) shows the same slice with ring removal applied.

Table 2

Comparative list of the four freeware programs outlining their available data-processing components.

\begin{tabular}{|c|c|c|c|c|c|c|c|}
\hline Program & Required platform & $\begin{array}{l}\text { Dark-field } \\
\text { calibration }\end{array}$ & $\begin{array}{l}\text { Flat-field } \\
\text { calibration }\end{array}$ & $\begin{array}{l}\text { Beam } \\
\text { normalization }\end{array}$ & Alignment & $\begin{array}{l}\text { Beam hardening } \\
\text { correction }\end{array}$ & $\begin{array}{l}\text { Ring artifact } \\
\text { correction }\end{array}$ \\
\hline Athabasca Recon & Windows Powershell & Yes & Yes & Yes & No & No & No \\
\hline NRecon & None & No & No & No & Yes & Yes & Yes \\
\hline H-PITRE & IDL Virtual Machine & Yes & Yes & Yes & Yes & No & Yes \\
\hline
\end{tabular}


The reconstruction of the dataset set can be completed individually or queued into batch jobs. Reconstructed images can be saved in various formats including TIFF, JPEG and BMP.

NRecon offers some advanced settings to improve image quality. The FBP algorithm can be adjusted for use with undersampled datasets, when objects are larger than the field of view, and for defective pixel masking. Multiple smoothing algorithms are available which alter each pixel on the projection images with a $M \times N$ (horizontal $\times$ vertical dimension, respectively) neighbourhood using either a box symmetric, box asymmetric or Gaussian kernel (Skyscan, 2011). The dynamic range of the image histogram may be edited to allow image enhancement.

NRecon has a useful manual and extensive help section; however, there is a lack of manual control to alter algorithms to optimize reconstruction precision as can be accomplished when using more direct algorithm application methods. NRecon is a user-friendly program which edits images quickly and efficiently and has an intuitive interface, but lacks features needed for many synchrotron-based datasets such as darkfield or flat-field correction and beam intensity normalization.

\subsection{PITRE}

PITRE (Phase-sensitive X-ray Image processing and Tomography REconstruction) can process data from highenergy X-ray source modalities including PC-CT, diffractionenhanced imaging processing, and phase retrieval for additional PC-CT data processing (Chen et al., 2012a). PITRE accepts a number of file types including 8-, 16- and 32-bit TIFF, 8-bit PNG and 8-bit BMP. PITRE uses the CPU of the workstation rather than the GPU and so has a slower reconstruction time than NRecon and H-PITRE which use the GPU. With no pre- or post-processing for our test dataset, reconstruction took approximately $4 \mathrm{~h}$ using PITRE (Table 1). PITRE_BM, a batch manager program that is provided with PITRE, allows maximization of CPU usage.

For in-line PC-CT reconstruction the 'Projection to Slice' data processing function was used. The other processes available are for other synchrotron-based data such as diffraction enhanced imaging. Prior to loading data one can choose between four methods used to calibrate images for dark-field and flat-field correction depending on whether the dark and flat images were taken before, after or during data acquisition, and this calibration occurs as the data is loaded. PITRE then converts the projections into sinograms for filtering and image processing. A single sinogram can be produced to create preview slices and define the main parameters (alignment, normalization and ring artifact removal) prior to producing all of the sinograms and completing the reconstruction. There are two reconstruction algorithms available to use: FBP (Kak \& Slaney, 2001) and Gridrec (Dowd et al., 1999). Gridrec improves computational efficiency with negligible artifacts through the use of the gridding method for resampling the Fourier space from polar to Cartesian coordinates (Marone \& Stampanoni, 2012). Fig. 4 shows output images with the Gridrec algorithm (Fig. 4a) and

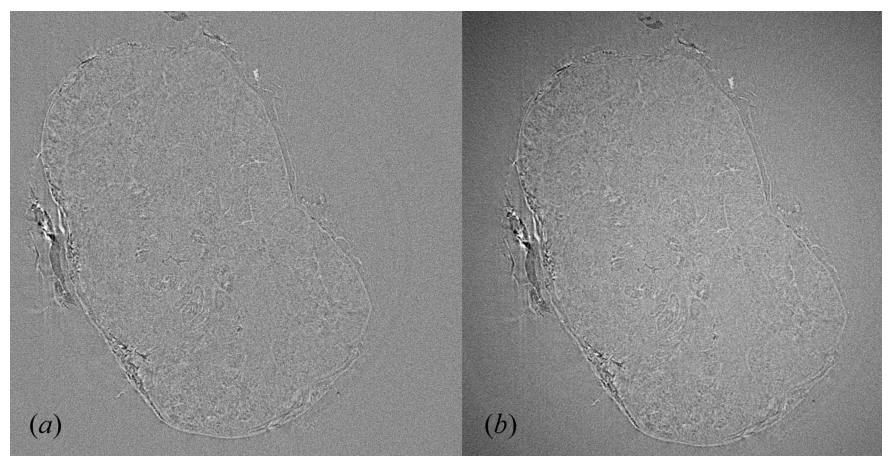

Figure 4

Reconstruction of the PC-CT data by (a) PITRE with the Gridrec algorithm, (b) PITRE with the FBP algorithm.

the FBP algorithm (Fig. 4b). PITRE provides an alignment tool that defines the centre of rotation of the sinograms by their pixel coordinates. A dynamic aligning method can also be used if there were shifts of the vertical axis during data collection. The dynamic adjustment extrapolates the centre of rotation for each sinogram by using pixel coordinates of the centre of rotation for the first and last sinogram. Beam intensity normalization is available whereby a range of projections are used to calculate and normalize an intensity curve which is then applied to the dataset. PITRE also provides a ring-removal algorithm that acts on the sinograms with an adjustable filter width. The algorithm finds the value of an average row of the sinogram by summing down each column and dividing by the number of rows, subtracts a smoothed version of this row to identify detector anomalies, and then subtracts these anomalies from each row of the sinogram (Chen et al., 2012a).

PITRE also offers a number of additional reconstruction options with a choice of window filters to be used with either the FBP or Gridrec algorithm, including Shepp-Logan, Hanning, Hamm, Ram-bak and Logarithm, which can be used to make data more uniform, reduce outlier pixels and reduce image noise. When a dataset is loaded, PITRE automatically defaults to the optimal filter based on the type of dataset. An extended view option is also included in this program which uses a dataset acquired over $360^{\circ}$ of rotation rather than $180^{\circ}$ to allow for the field of view to be increased. The program also allows conversion of 12-bit TIFF images to 16-bit TIFF format using a GraphicsMagick plugin. This is a valuable plugin as many detectors produce images in compressed 12-bit TIFF format rather than 16-bit to reduce imaging acquisition time.

PITRE is a useful detailed program that provides the ability for acute adjustments to increase the quality of volume slices; however, the program can be difficult to work with for an inexperienced user. The manual and the associated journal article are helpful, but lack explanations on how certain inputs should be defined. For example, it is not explicitly outlined as to what the units are for certain parameters. User experience could be improved with the addition of an expanded user's manual, but, regardless, there is no need for additional programs or plugins and there are a large number of manual adjustment options. 


\subsection{H-PITRE}

H-PITRE (High performance Phase-sensitive X-ray Image processing and tomography REconstruction) provides a graphic user interface which is very similar to PITRE but is designed for use with in-line PC-CT datasets only (Chen et al., $2012 b$ ). It is almost the same program with a few extra features devoted to PC-CT and all other features removed to create a simpler more efficient program. The actual set-up and functionality of the program are almost identical to PITRE with a main 'Projection to Sinogram' window and a window for viewing the reconstructed slices. The program accepts 8-, 16and 32-bit TIFF, PNG and BMP files with a naming index and produces 32-bit TIFF images. The program makes use of the GPU of the computer to increase the efficiency and speed of the reconstruction. This does result in strict computer requirements including: a $\mathrm{PC}$ with at least Windows $\mathrm{XP}$, an NVidia video card and a large RAM size to accommodate the sinogram production. Without any pre- or post-processing the reconstructions of the test dataset took approximately $1 \mathrm{~h}$ on the workstation used in this study (Table 1).

H-PITRE offers the same flat and dark calibration options, alignment method, normalization process, and ring artifact removal algorithm as PITRE and provides only the FBP algorithm to reconstruct data. Similar to PITRE, H-PITRE offers some advanced features such as an extended view option and window filters. An additional logarithmic filter is available in H-PITRE compared with PITRE which can be used alongside the other window filters. A region of interest can also be defined to crop the images, which can reduce reconstruction time and increase efficiency. PITRE_BM also works for $H$-PITRE to provide memory management for increased productivity. Overall the program is very similar to PITRE with a few extra features devoted to PC-CT and all other features removed to create a simpler more efficient program.

Overall $H$-PITRE is an excellent program for parallel-beam PC-CT. Its ability to use the GPU increases speed compared with the standard CPU version in PITRE. The program allows great freedom to alter parameters for optimal reconstructions. As the program is in its beta stage, the user's manual lacks details and explanations which can cause difficulty for inexperienced users.

\subsection{Athabasca Recon}

Athabasca Recon, version 1.3, was designed by the Bone Imaging Laboratory at the University of Calgary to work with parallel beam synchrotron tomographic data and provides all required features and data modifications needed to create good quality images. The program runs on a Windows Powershell command interface and uses ImageJ plug-ins to perform a number of tasks. This program does not have a graphic user interface, but is relatively simple to use. Athabasca Recon requires ITK MetaImage file (mhd) format to handle image sequences and uses an ImageJ plugin to create these files. The program allows access to some memory management settings, but the lack of GPU usage for compu- tations makes reconstructions slower. Reconstruction of the test dataset took approximately $6 \mathrm{~h}$.

The reconstruction is defined using configuration text files that outline the parameters chosen to run and build a reconstructed set of slices with Athabasca Recon. A first configuration file is used to complete dark- and flat-field correction, bad pixel removal, and variable beam power normalization while the second configuration file applies the $F B P$ algorithm. The Beam Power Correction has four different methods for correction depending on the dataset used. Alignment to the centre of rotation is completed by an ImageJ plugin, the Align Projections Tool. This tool was used on the test data following the application of the first configuration file. Ring artifact removal is not provided by Athabasca Recon so post-processing with additional software is needed to accomplish this task.

Athabasca Recon includes some advanced parameters to improve reconstruction quality. Bad Pixel Correction is a filter which identifies bad pixels and replaces them with the average value of the four nearest neighbours. Also available are smoothing filters with definable filter radius (Gaussian or tapered cosine filters), and a low-pass filter (removes high frequency noise). Athabasca Recon allows a choice in the method of pixel interpolation including nearest neighbour (less accurate; uses the value of the nearest pixel), bi-linear interpolation (interpolation from the four nearest pixels' centres) and bi-linear interpolation with fall back (used for volume data near to the edges of the projection; bi-linear interpolation loses a half pixel width of information so the edges are extrapolated using nearest neighbour). Lastly, Athabasca Recon allows for the threshold level of the darkfield and flat-field correction to be defined which allows for any pixel below the base flat-field threshold and above the dark-field threshold to be ignored.

Athabasca Recon provides control over the parameters of reconstruction through variation in the configuration file. The manual and the program are both very explicit on the technical details allowing a better understanding of how the data are manipulated. However, the lack of a graphic user interface can be challenging to those with less exposure to commandline interface programs. The usability of the program is further reduced as various parameters must be chosen prior to reconstruction with no available method to preview the slices. Overall, Athabasca Recon may be appealing due to the availability of a variety of filters and settings for fine-tuning the reconstructions. Slow computational speed, lack of a GUI and no ring artifact removal feature are its major limitations.

Images created using H-PITRE $(21.37 \mathrm{~dB})$ and NRecon (20.23 dB) provided the highest PSNR when compared with the reference image, while PITRE $(18.71 \mathrm{~dB})$ and Athabasca Recon $(19.05 \mathrm{~dB})$ provided the lowest. When compared with the reference image the SSIM was the highest with PITRE (0.26) followed by NRecon (0.25), H-Pitre (0.23) and Athabasca Recon (0.18). PITRE, NRecon and H-PITRE thus have very close structural similarity indices, whereas Athabasca Recon has lower similarity with the reference image which is not surprising considering that Athabasca Recon does not have native ring removal. 


\section{Discussion}

All of the programs reviewed are useful tools which should be considered for their benefits and disadvantages when used for a specific dataset. NRecon is a good general-purpose program with a user-friendly interface with a well written user manual and help menu, but compared with other programs provides limited control over reconstruction settings. Both PITRE and H-PITRE are excellent programs; however, their user manual, help menu and user interface were not explicit and many of the finer details of the programs had to be discovered by trial and error. PITRE is a useful program that provides options for the majority of reconstruction parameters. H-PITRE is almost the same as PITRE except it is simplified for a single purpose (PC-CT), rather than having multiple functions. Athabasca Recon has a command-based rather that graphic-based user interface which can make it a difficult program for some users. Athabasca Recon has a detailed user manual which explains all of the processes and tools.

The four programs offered similar tools with their availability listed in Table 2. For our test dataset, H-PITRE and NRecon provided the highest PSNRs, though H-PITRE, NRecon and PITRE had very similar SSIMs and provide very comparable images subjectively (Fig. 3). NRecon lacks the ability to normalize the beam power over time. For our dataset, which was gathered within less than $1 \mathrm{~h}$, projections calibrated using NRecon (without beam power normalization) and Athabasca Recon (with normalization) showed no significant difference between the first $\left(\right.$ at $\left.0^{\circ}\right)$ and the final (at $180^{\circ}$ ) projection. For data imaged over a longer period this may become a considerable problem and beam normalization would be an important feature.

The use of the GPU greatly increases reconstruction speed even on a single machine which allows for large datasets to be dealt with efficiently. Neither PITRE nor Athabasca Recon use the GPU for computations, and the reconstructions both took more than $4 \mathrm{~h}$ (Table 1) for our test dataset, whereas NRecon and H-PITRE, which use the GPU, completed the task within $1 \mathrm{~h}$. Athabasca Recon took six times as long as NRecon to complete reconstructions. NRecon and H-PITRE are better suited programs for generating three-dimensional stacks of images for surface or volume rendering where efficiency of reconstruction (i.e. the reconstruction time) is an important determining factor. Software that uses the GPU of the system is generally better suited to processing large PC-CT data sets and should be taken into consideration when choosing a system.

\section{Conclusion}

The use of in-line PC-CT is rapidly growing as a valuable imaging modality. Like other modalities, usefulness and speed of this method for medical diagnostic or research purposes depends on the resources required to generate images such as the physical facility to collect data, powerful computer hardware for processing, and versatile software to manipulate, extract and analyze information from the raw data. This review examined four free software programs (NRecon, PITRE, H-PITRE, Athabasca Recon) for their ease of use, computation speed and efficiency, high-quality reconstruction algorithms, degree of manual/user control, and corrections for image quality. By defining the needs with regards to usability, efficiency and availability of features, users can easily choose a program from this list which will adequately meet their needs.

\section{Acknowledgements}

Funding for this research was provided by the Saskatchewan Health Research Foundation, and Ride for Dad - Prostate Cancer Fight Foundation. Research described in this paper was performed at the Canadian Light Source, which is funded by the Canada Foundation for Innovation, the Natural Sciences and Engineering Research Council of Canada, the National Research Council Canada, the Canadian Institutes of Health Research, the Government of Saskatchewan, Western Economic Diversification Canada, and the University of Saskatchewan.

\section{References}

Bourland, J. D. (2012). Image-Guided Radiation Therapy. Boca Raton: CRC Press.

Bravin, A., Coan, P. \& Suortti, P. (2013). Phys. Med. Biol. 58, R1-R35.

Burvall, A., Lundström, U., Takman, P. A., Larsson, D. H. \& Hertz, H. M. (2011). Opt. Express, 19, 10359-10376.

Chen, R.-C., Dreossi, D., Mancini, L., Menk, R., Rigon, L., Xiao, T.-Q. \& Longo, R. (2012a). J. Synchrotron Rad. 19, 836-845.

Chen, R.-C., Dreossi, D., Mancini, L., Menk, R., Rigon, L., Xiao, T. \& Longo, R. (2012b). H-PITRE, http://webint.ts.infn.it/en/research/ Exp/beats2/h-pitre-beta-version.html.

Chen, R.-C., Dreossi, D., Mancini, L., Menk, R., Rigon, L., Xiao, T. \& Longo, R. (2013a). PITRE version 3.1, http://webint.ts.infn.it/en/ research/Exp/beats2/pitre.html.

Chen, R.-C., Dreossi, D., Mancini, L., Menk, R., Rigon, L., Xiao, T. \& Longo, R. (2013b). PITRE Manual, http://webint.ts. infn.it/fileadmin/Int/physics/experiments/beats2/pitre/PITRE_ Manual.pdf.

Chen, R., Xie, H., Rigon, L., Du, G., Castelli, E. \& Xiao, T. (2010). Tinshhua Sci. Technol. 15, 102-107.

Deák, Z., Grimm, J. M., Treitl, M., Geyer, L. L., Linsenmaier, U., Körner, M., Reiser, M. F. \& Wirth, S. (2013). Radiology, 266, 197206.

Diemoz, P., Bravin, A., Langer, M. \& Coan, P. (2012). Opt. Express, 20, 27670-27690.

Dowd, B. A., Campbell, G. H., Marr, R. B., Nagarkar, V. V., Tipnis, S. V., Axe, L. \& Siddons, D. P. (1999). Proc. SPIE, 3772, 224-236.

Eklund, A., Dufort, P., Forsberg, D. \& LaConte, S. M. (2013). Med. Image Anal. 17, 1073-1094.

Flores, L. A., Vidal, V., Mayo, P., Rodenas, F. \& Verdú, G. (2013). Proc. Comput. Sci. 18, 1412-1420.

Guo, X., Liu, X., Gu, M., Ni, C., Huang, S. \& Liu, B. (2012). Europhys. Lett. 98, 14001.

Herman, G. T. (2010). Fundamentals of Computerized Tomography: Image Reconstruction from Projections, 2nd ed. London: Springer.

Kak, A. C. \& Slaney, M. (2001). Principles of Computerized Tomographic Imaging. Philadelphia: Society for Industrial and Applied Mathematics.

Kastner, J., Harrer, B., Requena, G. \& Brunke, O. (2010). NDT Intl, 43, 599-605.

Lewis, R. (1997). Phys. Med. Biol. 42, 1213-1243.

Lewis, R. (2004). Phys. Med. Biol. 49, 3573-3583. 
Marone, F. \& Stampanoni, M. (2012). J. Synchrotron Rad. 19, 10291037.

Meuli, R., Hwu, Y., Je, J. H. \& Margaritondo, G. (2004). Eur. Radiol. 14, 1550-1560.

Mohammadi, S., Brun, F., Dullin, C., Dreossi, D. \& Tromba, G. (2013). 2013 8th International Symposium on Image and Signal Processing and Analysis (ISPA), pp. 622-626. IEEE.

Radon, J. (1986). IEEE Trans. Med. Imaging, 5, 170-176.

Schiwietz, T., Chang, T.-C., Speier, P. \& Westermann, R. (2006). Proc. SPIE, 6142, 61423T.

Singh, S., Kalra, M. K., Hsieh, J., Licato, P. E., Do, S., Pien, H. H. \& Blake, M. A. (2010). Radiology, 257, 373-383.

Skyscan (2011). NRecon User Manual, http://www.skyscan.be/next/ NReconUserGuide.pdf.

Stock, S. R. (2009). MicroComputed Tomography Methodology and Applications. Boca Raton: CRC Press.

Suortti, P. \& Thomlinson, W. (2003). Phys. Med. Biol. 48, R1-R35.
Thomlinson, W., Suortti, P. \& Chapman, D. (2005). Nucl. Instrum. Methods Phys. Res. A, 543, 288-296.

Van de Casteele, E., Van Dyck, D., Sijbers, J. \& Raman, E. (2002). Phys. Med. Biol. 47, 4181-4190.

Vo, N. T., Atwood, R. C., Moser, H. O., Lee, P. D., Breese, M. B. \& Drakopoulos, M. (2012). Appl. Phys. Lett. 101, 224108.

Wang, Z., Bovik, A. C., Sheikh, H. R. \& Simoncelli, E. P. (2004). IEEE Trans. Image Process. 13, 600-612.

Weitkamp, T., Haas, D. \& Rack, A. (2014). ANKAPhase, http:// rsb.info.nih.gov/ij/plugins/ankaphase/.

Weitkamp, T., Haas, D., Wegrzynek, D. \& Rack, A. (2011). J. Synchrotron Rad. 18, 617-629.

Wu, X., Liu, H. \& Yan, A. (2008). Eur. J. Radiol. 68, S8-S12.

Wysokinski, T. W., Chapman, D., Adams, G., Renier, M., Suortti, P. \& Thomlinson, W. (2007). Nucl. Instrum. Methods Phys. Res. A, $73-$ 76.

Zhou, S.-A. \& Brahme, A. (2008). Phys. Med. 24, 129-148. 